\title{
$Z Z$ production at the LHC: NLO QCD corrections to the loop-induced gluon fusion channel
}

\author{
Massimiliano Grazzini, ${ }^{a}$ Stefan Kallweit, ${ }^{b, c}$ Marius Wiesemann ${ }^{b}$ and Jeong Yeon Yook $^{a}$ \\ ${ }^{a}$ Physik-Institut, Universität Zürich, \\ Winterthurerstrasse 190, CH-8057 Zürich, Switzerland \\ ${ }^{b}$ TH Division, Physics Department, CERN, \\ CH-1211 Geneva 23, Switzerland \\ ${ }^{c}$ Università degli Studi di Milano-Bicocca, \\ Piazza della Scienza 3, I-20126 Milan, Italy \\ E-mail: grazzini@physik.uzh.ch, stefan.kallweit@cern.ch, \\ marius.wiesemann@cern.ch, jyook@physik.uzh.ch
}

ABSTRACT: We consider QCD radiative corrections to the production of four charged leptons in hadron collisions. We present the computation of the next-to-leading order QCD corrections to the loop-induced gluon fusion contribution. Our predictions include, for the first time, also the quark-gluon partonic channels. The computed corrections, which are formally of $\mathcal{O}\left(\alpha_{\mathrm{S}}^{3}\right)$, turn out to increase the loop-induced Born-level result by an amount ranging from $75 \%$ to $71 \%$ as $\sqrt{s}$ ranges from 8 to $13 \mathrm{TeV}$. We combine our result with state-of-the-art NNLO corrections to the quark annihilation channel, and present updated predictions for fiducial cross sections and distributions for this process.

KeYwords: NLO Computations, QCD Phenomenology

ARXIV EPRINT: 1811.09593 


\section{Contents}

1 Introduction 1

2 Calculation within the MATRIX framework 2

3 Validation 4

4 Results $\quad 6$

4.1 Setup 6

$\begin{array}{lll}4.2 & \text { Fiducial cross section and distributions } & 7\end{array}$

$\begin{array}{lll}5 & \text { Summary } & 14\end{array}$

\section{Introduction}

The production of $Z$-boson pairs is one of the most relevant processes at the Large Hadron Collider (LHC). Besides providing an important test of the electroweak (EW) sector of the Standard Model (SM) at the TeV scale, it was instrumental for the discovery of the Higgs boson [1, 2]. As the focus of Higgs physics moved from the discovery to the study of its properties, $Z$-boson pair production played an essential role in the determination of the quantum numbers of the new resonance [3, 4], in setting bounds on its width (see e.g. refs. [5, 6]), and in constraining anomalous Higgs boson couplings [7].

At the leading order (LO) in the QCD coupling $\alpha_{\mathrm{S}}, Z$-boson pairs are produced via quark annihilation. Theoretical predictions for $Z Z$ production at next-to-leading order (NLO) QCD were obtained a long time ago for both on-shell $Z$ bosons [8, 9] and their fully leptonic final states [10-13]. Perturbative corrections beyond NLO QCD are indispensable to reach the precision demanded by present $Z Z$ measurements. NLO EW corrections are known for stable $Z$ bosons [14-16] and including their leptonic decays with full off-shell effects $[17,18]$. NLO QCD $+\mathrm{EW}$ results for the $2 \ell 2 \nu$ signature have been presented in ref. [19], and for the $\ell \ell \ell^{\prime} \ell^{\prime}$ signature with the inclusion of anomalous couplings in ref. [20]. $Z Z+$ jet production was computed at NLO QCD [21]. The loop-induced gluon fusion channel, which provides a separately finite $\mathcal{O}\left(\alpha_{\mathrm{S}}^{2}\right)$ contribution and is enhanced by the large gluon luminosity, has been known at LO for a long time [22-32]. It was recently computed at NLO [33-35] using the two-loop helicity amplitudes for $g g \rightarrow V V^{\prime}$ of refs. [36, 37], considering only the gluon-gluon $(g g)$ partonic channel. NNLO QCD corrections to onshell $Z Z$ production were first evaluated in ref. [38], and later in ref. [39]. Using the two-loop helicity amplitudes for $q \bar{q} \rightarrow V V^{\prime}$ [40-42], fully differential NNLO predictions in the four-lepton channels ( $\ell \ell \ell \ell$ and $\ell \ell \ell^{\prime} \ell^{\prime}$ ) were first presented in ref. [43], while in ref. [44] also the $2 \ell 2 \nu$ signature was considered. 


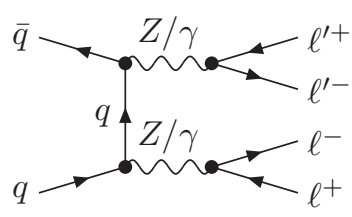

(a)

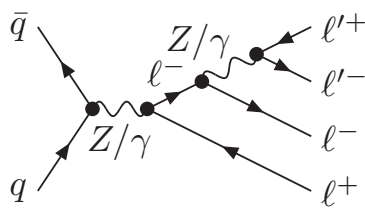

(b)

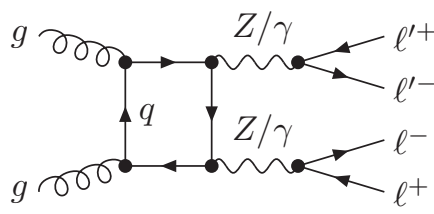

(c)

Figure 1. Sample Feynman diagrams for $Z Z$ production with four charged final-state leptons: tree-level diagrams of the quark annihilation channel in (a) and (b), loop-induced diagram of the gluon fusion channel in (c).

An analogous situation is the one of $W^{+} W^{-}$production, for which NNLO QCD corrections $[45,46]$ to quark annihilation are available, and NLO QCD corrections to the loopinduced gluon fusion contribution were computed recently [47]. At present, experimental analyses for both $Z Z$ and $W W$ production treat the quark annihilation and loop-induced gluon fusion channels as if they were independent. As a result, data are compared to ad hoc combinations of NNLO calculations for the quark annihilation channel and NLO calculations for the loop-induced gluon fusion channel, often by using $K$-factors (see e.g. refs. [48-51]). However, it is well known that the quark-antiquark $(q \bar{q})$ and $g g$ partonic channels mix through parton evolution, and thus their independent treatment is not appropriate. Moreover, already at NNLO there are diagrams that mix the two production mechanisms, thereby suggesting that a unified treatment would be desirable. This is particularly important to consistently estimate the perturbative uncertainties through variation of the renormalisation and factorisation scales.

In this paper we take a decisive step in this direction, by combining the NNLO calculation in the quark annihilation channel with the NLO calculation of the loop-induced gluon fusion channel. For the first time, we also evaluate the (anti)quark-gluon $(q g)$ contributions that enter the full NLO corrections to the loop-induced channel. We introduce an approximation of the full $\mathrm{N}^{3} \mathrm{LO}$ corrections, denoted by "nNNLO", which represents the most advanced perturbative QCD prediction available at present for this process. The new calculation will be available in an updated version of MATRIX [52].

The paper is organised as follows. In section 2 we introduce our computational framework. In section 3 we present a comparison of our results to those of ref. [35]. In section 4 we combine our computations of radiative corrections to the quark annihilation and loopinduced gluon fusion channels, and present fiducial cross sections and distributions in $p p$ collisions at 8 and $13 \mathrm{TeV}$. In section 5 we summarise our results.

\section{Calculation within the MATRIX framework}

We consider the four-lepton process

$$
p p \rightarrow \ell^{+} \ell^{-} \ell^{\prime+} \ell^{\prime-}+X,
$$

where, for simplicity, we assume the triggered lepton pairs to have different flavours $(\ell \neq$ $\ell^{\prime}$ ). Representative Born-level diagrams are shown in figure 1. Diagrams (a) and (b) are 


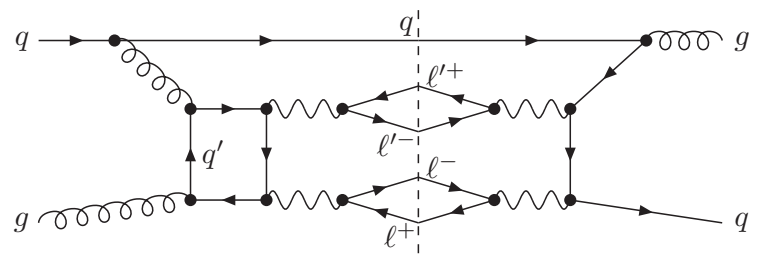

Figure 2. Example of NNLO interference between quark annihilation and loop-induced gluon fusion production mechanisms.

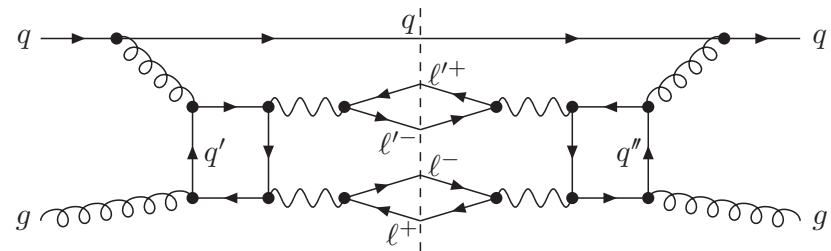

(a)

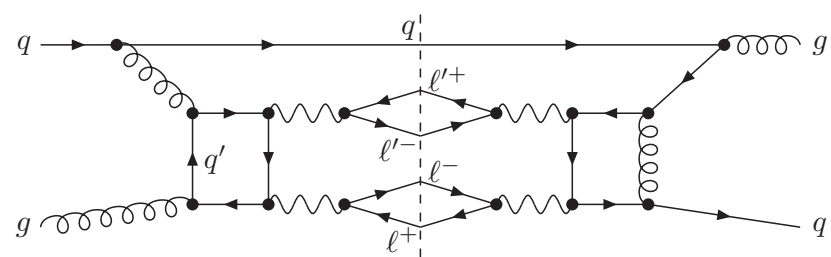

(b)

Figure 3. Examples of $\mathrm{N}^{3} \mathrm{LO}$ contributions in the $q g$ channel.

driven by quark annihilation and show double-resonant $t$-channel $Z Z$ production and singleresonant $s$-channel Drell-Yan topologies, respectively. Diagram (c) is instead driven by gluon fusion through a quark loop, and it enters the calculation at NNLO as it is of $\mathcal{O}\left(\alpha_{\mathrm{S}}^{2}\right)$. However, this contribution is enhanced by the large gluon luminosity. Up to NLO the quark annihilation and loop-induced gluon fusion production processes do not mix. Until a few years ago, the theoretical standard was to consider NLO-accurate predictions for the quark annihilation channel, supplemented with the loop-induced gluon fusion contribution [27].

Starting from NNLO, the quark annihilation and loop-induced gluon fusion processes mix, and the distinction between the two production mechanisms is questionable. An example of an interference contribution is shown in figure 2. A complete NNLO computation of four-lepton production has been presented in refs. [43, 44]. At this order, the loopinduced gluon fusion contribution enters the cross section through the square of diagrams like the one in figure $1(\mathrm{c})$. The fact that this $\mathcal{O}\left(\alpha_{\mathrm{S}}^{2}\right)$ contribution is quite large and formally only LO accurate motivates the inclusion of NLO corrections to the loop-induced gluon fusion channel, which are part of the $\mathrm{N}^{3} \mathrm{LO}$ corrections. We point out that the loopinduced contributions are not the only contributions to the $g g$ channel at $\mathrm{N}^{3} \mathrm{LO}$. However, we expect the impact of the $\mathrm{N}^{3} \mathrm{LO}$ non-loop-induced diagrams to be within the perturbative uncertainties estimated by studying scale variations at NNLO. The same cannot be said for the loop-induced contributions. The NLO computation for a loop-induced process 
requires one-loop amplitudes with the emission of one additional parton and two-loop contributions. In refs. $[33,35]$ the calculation has been performed by considering only the $g g$ partonic channel. Here we extend the above calculation by including also the $q g$ initiated contributions. ${ }^{1}$ We note that at $\mathrm{N}^{3} \mathrm{LO}$ we only include diagrams with closed fermion loops (see figure $3(\mathrm{a})$ ); all other contributions that would enter a complete $\mathrm{N}^{3} \mathrm{LO}$ calculation (see figure 3 (b) for example) cannot be consistently accounted for at present. Our approximation includes all contributions at $\mathcal{O}\left(\alpha_{\mathrm{S}}^{2}\right)$ together with the complete NLO corrections to the loop-induced gluon fusion channel at $\mathcal{O}\left(\alpha_{\mathrm{S}}^{3}\right)$. As such, besides providing the maximum perturbative information available at present for this process, our calculation can be used to obtain a consistent estimate of perturbative uncertainties through the customary procedure of studying scale variations.

Our calculation is carried out within the computational framework MATRIX [52]. MATRIX features a fully general implementation of the $q_{T}$-subtraction formalism [53] and allowed us to compute NNLO QCD corrections to a large number of colour-singlet processes at hadron colliders $[38,43,45,46,54-59] .{ }^{2}$ The core of the MATRIX framework is the Monte Carlo program MUNICH, which is capable of computing both NLO QCD and NLO EW $[62,63]$ corrections to arbitrary SM processes. ${ }^{3}$

As in previous MATRIX calculations, in our computation of the NLO corrections to the $g g \rightarrow 4 \ell$ process, all the required one-loop amplitudes are evaluated with OpenLoops ${ }^{4}[70$, 71]. To the purpose of validating our results for the loop-induced contribution, we have used also the independent matrix-element generator RECOLA [72, 73], finding complete agreement.

At two-loop level, we use the $g g \rightarrow V V^{\prime}$ helicity amplitudes of ref. [37], and implement the corresponding four-lepton final states, accounting for spin correlations and off-shell effects. The NLO calculation is performed by using the Catani-Seymour dipole-subtraction method $[74,75]$ and also with $q_{T}$ subtraction [53], which provides an additional cross-check of our results.

\section{Validation}

The NLO corrections to the loop-induced gluon fusion contribution have been first computed in refs. [33, 35], where the $q g$ partonic channels were neglected. The results of ref. [33] are provided with only two significant digits, and without any information on numerical or systematic uncertainties. Although we were able to reproduce their results at this level of

\footnotetext{
${ }^{1}$ We note that there are also $q \bar{q}$ initiated contributions to the loop-induced production mechanism at $\mathcal{O}\left(\alpha_{\mathrm{S}}^{3}\right)$, which are separately finite. We found them to be completely negligible and ignore them in the following. Our results include all numerically relevant partonic channels of the NLO corrections to the loop-induced gluon fusion contribution.

${ }^{2}$ It was also used in the NNLL+NNLO computation of ref. [60], and in the NNLOPS computation of ref. [61].

${ }^{3} \mathrm{MUNICH}$ is the abbreviation of "MUlti-chaNnel Integrator at Swiss (CH) precision". See ref. [64].

${ }^{4}$ OpenLoops relies on the fast and stable tensor reduction of Collier [65, 66], supported by a rescue system based on quad-precision CutTools [67] with OneLOop [68] to deal with exceptional phase-space points. All relevant loop-induced amplitudes with correlators will be available in an upcoming publication of OpenLoOps2 [69].
} 
precision, they are not particularly suitable for a detailed comparison. More precise results are stated in ref. [35], and with the statistical errors provided in private communication a reasonable technical comparison could be performed. The calculations in refs. [33, 35] are carried out by using five massless quark flavours $\left(N_{f}\right)$, and the contributions of both top-quark loops and triangle diagrams are omitted. ${ }^{5}$ Furthermore, the $q g$ initiated subprocesses were neglected. The loop-induced gluon fusion contribution (denoted by $g g \mathrm{LO}$ in the following) and its NLO corrections restricted to the $g g$ partonic channel (denoted by $g g \mathrm{NLO}_{g g}$ in the following) are both computed with $N_{f}=5$ NNPDF3.0 [76] NLO parton distribution functions with $\alpha_{\mathrm{S}}\left(m_{Z}\right)=0.118$.

For validation, we have repeated the calculation of ref. [35], using exactly the same setup: cuts are only applied on the invariant mass of the opposite-sign same-flavour (OSSF) lepton pairs $\left(m_{\ell^{+} \ell^{-}}\right)$and on the four-lepton invariant mass $\left(m_{4 \ell}\right)$, which are required to fulfil

$$
5 \mathrm{GeV}<m_{\ell^{+} \ell^{-}}<180 \mathrm{GeV}, \quad 60 \mathrm{GeV}<m_{4 \ell}<360 \mathrm{GeV} .
$$

The comparison of our results to those in table I of ref. [35] (denoted by ACLR) is reported in table 1. Both settings of ref. [35] for the renormalisation $\left(\mu_{R}\right)$ and factorisation $\left(\mu_{R}\right)$ scales are considered: fixed values $\mu_{R}=\mu_{F}=\mu_{0} \equiv m_{Z}$, with $m_{Z}$ being the $Z$-boson mass, and dynamical values $\mu_{R}=\mu_{F}=\mu_{0} \equiv m_{4 \ell} / 2$. The quoted uncertainties are estimated from customary 7-point scale variations by a factor of two, with the constraint $0.5 \leq \mu_{R} / \mu_{F} \leq 2$. For both our and the ACLR results we provide statistical errors on the respective last digit(s) inside parentheses.

Taking into account the numerical errors of the ACLR results, which are typically of comparable size as our errors, we find that all $8 \mathrm{TeV}$ results are in perfect statistical agreement, corresponding to discrepancies of only a few per mille of the respective $g g \mathrm{NLO}_{g g}$ predictions. The agreement between the $g g \mathrm{NLO}_{g g}$ results at $13 \mathrm{TeV}$ turns out to be slightly worse: while the exceptionally large discrepancy of $-2.1 \%$ with $\mu_{0}=m_{Z}$ as central scale choice is fully covered by the statistical ACLR error within one standard deviation, the few-permille discrepancy in the result for $\mu_{0}=m_{4 \ell} / 2$ corresponds to a discrepancy of three standard deviations. We note that the calculation of ref. [35] uses a technical cut $p_{T}^{Z Z}>0.5 \mathrm{GeV}$ on the transverse momentum of the four-lepton system, in order to avoid instabilities in the one-loop matrix elements. Also our calculation involves technical cuts based on parameters controlling the minimum invariant masses of parton pairs and the internal stability estimate of the OpEnLoops amplitudes. By varying the above technical parameters we estimate the systematic uncertainties affecting our NLO results in table 1 to be at the few-permille level. Indeed, this level of precision is confirmed by our alternative implementation using $q_{T}$ subtraction. This additional source of uncertainties in the two predictions could explain the slight discrepancy we observe in the NLO result at $13 \mathrm{TeV}$ with $\mu_{0}=m_{4 \ell} / 2$, which is poorly covered by the statistical errors only. We stress that for the purpose of phenomenological applications all the observed differences are subleading

\footnotetext{
${ }^{5}$ The fermionic triangle diagrams vanish in the sum over a massless quark generation. Correspondingly, neglecting the remaining bottom-quark contribution is a reasonable ansatz if the top-quark contribution cannot be taken into account.
} 


\begin{tabular}{|c|c|c|c|c|c|}
\hline & & \multicolumn{2}{|c|}{$\mu=m_{4 l} / 2$} & \multicolumn{2}{c|}{$\mu=m_{Z}$} \\
\hline$\sqrt{s}$ & & ggLO & ggNLO $_{g g}$ & ggLO & ggNLO $_{g g}$ \\
\hline $8 \mathrm{TeV}$ & ACLR & $1.6018(5)_{-0.30}^{+0.41}$ & $2.980(5)_{-0.41}^{+0.51}$ & $1.6181(6)_{-0.31}^{+0.42}$ & $2.978(4)_{-0.40}^{+0.496}$ \\
& MATRIX & $1.6023(4)_{-0.30}^{+0.41}$ & $2.987(3)_{-0.42}^{+0.51}$ & $1.6188(3)_{-0.31}^{+0.42}$ & $2.985(3)_{-0.40}^{+0.49}$ \\
\hline \multirow{2}{*}{$13 \mathrm{TeV}$} & ACLR & $3.8467(13)_{-0.70}^{+0.97}$ & $6.984(8)_{-0.94}^{+1.14}$ & $3.9429(14)_{-0.71}^{+0.98}$ & $7.22(18)_{-1.04}^{+1.04}$ \\
& MATRIX & $3.8486(8)_{-0.70}^{+0.97}$ & $7.016(7)_{-0.95}^{+1.15}$ & $3.9454(8)_{-0.71}^{+0.98}$ & $7.068(7)_{-0.93}^{+1.11}$ \\
\hline
\end{tabular}

Table 1. Comparison of our MATRIX results with those of ref. [35]. ${ }^{6}$

since a percent effect in the loop-induced gluon fusion channel only leads to a permille effect on the complete inclusive and fiducial four-lepton cross sections due to the dominance of the quark annihilation channel. We also note that we were able to qualitatively (on the level of the plots) reproduce all differential distributions shown in refs. [33, 35].

\section{Results}

\subsection{Setup}

We present predictions for $p p \rightarrow e^{+} e^{-} \mu^{+} \mu^{-}$production at 8 and $13 \mathrm{TeV}$. For the EW parameters we employ the $G_{\mu}$ scheme and set $\alpha=\sqrt{2} G_{\mu} m_{W}^{2}\left(1-m_{W}^{2} / m_{Z}^{2}\right) / \pi$. Contrary to section 3 , we compute the EW mixing angle as $\cos \theta_{W}^{2}=\left(m_{W}^{2}-i \Gamma_{W} m_{W}\right) /\left(m_{Z}^{2}-i \Gamma_{Z} m_{Z}\right)$ and use the complex-mass scheme [77] throughout. The EW inputs are set to the PDG [78] values: $G_{F}=1.16639 \times 10^{-5} \mathrm{GeV}^{-2}, m_{W}=80.385 \mathrm{GeV}, \Gamma_{W}=2.0854 \mathrm{GeV}, m_{Z}=$ $91.1876 \mathrm{GeV}, \Gamma_{Z}=2.4952 \mathrm{GeV}, m_{H}=125 \mathrm{GeV}$, and $\Gamma_{H}=0.00407$. The on-shell topquark mass is set to $m_{t}=173.2 \mathrm{GeV}$, and $\Gamma_{t}=1.44262$ is used. Except for virtual two-loop contributions, the full dependence on massive top quarks is taken into account everywhere in the computation. For the $q \bar{q} \rightarrow Z Z$ subprocess, the contribution of top quarks in the twoloop corrections is not known. Since the quantitative impact of the two-loop diagrams with a light fermion loop is extremely small [38], the two-loop diagrams involving a top-quark can be safely neglected. Disregarding Higgs boson exchange, ${ }^{7}$ top-quark loops contribute about $2 \%$ to the $g g \mathrm{LO}$ cross section and should thus be included. However, also for the $g g \rightarrow Z Z$ subprocess, the top-quark contribution in the two-loop amplitude is unknown. Here we approximate top-quark effects through a reweighting of the massless two-loop result by the LO (one-loop) amplitude with full top-quark mass dependence. Diagrams involving the Higgs boson are consistently included at each perturbative order, except for the two-loop contributions, where we employ the same approximation as for the massive top loops.

For each perturbative order we use the corresponding set of $N_{f}=5$ NNPDF3.0 [76] parton distribution functions (PDFs) with $\alpha_{\mathrm{S}}\left(m_{Z}\right)=0.118$. The loop-induced gluon fusion

\footnotetext{
${ }^{6} \mathrm{~A}$ typo in the upper scale variation of the $g g \mathrm{NLO}_{g g}$ result for $\sqrt{s}=8 \mathrm{TeV}, \mu_{0}=m_{4 \ell} / 2$ of ref. [35] has been corrected.

${ }^{7}$ Quantitative comments on the impact of Higgs boson diagrams are postponed to section 4.2.
} 


\begin{tabular}{|c|}
\hline definition of the fiducial volume for $p p \rightarrow e^{+} e^{-} \mu^{+} \mu^{-}+X$ \\
\hline$p_{T, e / \mu}>7 \mathrm{GeV}, \quad$ one electron with $\left|\eta_{e}\right|<4.9, \quad$ the others $\left|\eta_{e}\right|<2.5, \quad\left|\eta_{\mu}\right|<2.7$ \\
$\Delta R_{e e / \mu \mu}>0.2, \quad \Delta R_{e \mu}>0.2, \quad 66 \mathrm{GeV} \leq m_{e^{+} e^{-} / \mu^{+} \mu^{-}} \leq 116 \mathrm{GeV}$, \\
\hline
\end{tabular}

Table 2. Phase-space definitions of the $Z Z$ measurements by ATLAS at $8 \mathrm{TeV}$ [79].

contribution and its NLO corrections are always computed with NNLO PDFs. The renormalisation and factorisation scales are set to half of the invariant mass of the four-lepton system, $\mu_{R}=\mu_{F}=\mu_{0} \equiv m_{4 \ell} / 2$. Residual uncertainties are estimated from customary 7-point scale variations by a factor of two, with the constraint $0.5 \leq \mu_{R} / \mu_{F} \leq 2$.

We use the selection cuts adopted by the ATLAS collaboration at $8 \mathrm{TeV}$ in ref. [79], which are summarized in table $2 .{ }^{8}$ The fiducial cuts involve standard requirements on the transverse momenta and pseudo-rapidities of the leptons, a pair-wise separation in $\Delta R=\sqrt{\Delta \eta^{2}+\Delta \phi^{2}}$ between all possible leptons (independently of their flavours and charges), and a window in the invariant mass of reconstructed $Z$ bosons around the $Z$-pole.

\subsection{Fiducial cross section and distributions}

We briefly introduce the notation used throughout this section: the loop-induced gluon fusion channel contributes at $\mathcal{O}\left(\alpha_{\mathrm{S}}^{2}\right)$, and is denoted by $g g \mathrm{LO}$ in the following. The NNLO result for the quark annihilation channel, i.e. without the loop-induced contribution, is referred to as $q \bar{q}$ NNLO. The complete loop-induced contribution at NLO is labelled $g g$ NLO, while its restriction to the $g g$ partonic channel is dubbed as $g g \mathrm{NLO}_{g g}$, i.e. the difference between these two predictions corresponds to the newly computed contribution from the $q g$ channels. As discussed in the Introduction, the NLO corrections to the loop-induced contribution are only a part of the complete $\mathrm{N}^{3} \mathrm{LO}$ computation. However, these corrections are sizeable, and the loop-induced gluon fusion production mechanism is known to be only poorly described at $\mathcal{O}\left(\alpha_{\mathrm{S}}^{2}\right)$, namely at its effective LO. It is thus reasonable to construct an approximation of the complete $\mathrm{N}^{3} \mathrm{LO}$ cross section based on the inclusion of only these $\mathcal{O}\left(\alpha_{\mathrm{S}}^{3}\right)$ corrections. This approximation is denoted by nNNLO.

We present the fiducial cross sections for $\sqrt{s}=8$ and $13 \mathrm{TeV}$ at the various perturbative orders in table 3. In the upper panel the QCD corrections to the quark annihilation channel are reported. The NNLO corrections to this channel amount to about $+7 \%(+9 \%)$ at $\sqrt{s}=8(13) \mathrm{TeV}$. In the central panel the loop-induced gluon fusion contribution is shown with its NLO corrections. Comparing the results with NNLO PDFs used throughout, this contribution provides $57 \%(62 \%)$ of the full NNLO corrections at $\sqrt{s}=8(13) \mathrm{TeV}$. The NLO corrections increase the $g g \mathrm{LO}$ result by about $75 \%(71 \%)$ at $\sqrt{s}=8(13) \mathrm{TeV}$. The contribution of the $q g$ channels is negative, such that the cross section becomes about $7 \%(6 \%)$ larger wrt. $g g \mathrm{NLO}$ at $\sqrt{s}=8(13) \mathrm{TeV}$ if contributions from $q g$ partonic channels are neglected $\left(g g \mathrm{NLO}_{g g}\right)$. In the lower panel, the NNLO and nNNLO results are shown. The impact of the NLO corrections to the loop-induced contribution is to increase the

\footnotetext{
${ }^{8}$ For simplicity, we employ the same setup at $13 \mathrm{TeV}$.
} 


\begin{tabular}{|c|c|c|c|c|}
\hline$\sqrt{s}$ & $8 \mathrm{TeV}$ & $13 \mathrm{TeV}$ & $8 \mathrm{TeV}$ & $13 \mathrm{TeV}$ \\
\hline & \multicolumn{2}{|c|}{$\sigma[\mathrm{fb}]$} & \multicolumn{2}{|c|}{$\sigma / \sigma_{\mathrm{NLO}}-1$} \\
\hline LO & $8.1881(8)_{-3.2 \%}^{+2.4 \%}$ & $13.933(1)_{-6.4 \%}^{+5.5 \%}$ & $-27.5 \%$ & $-29.8 \%$ \\
\hline NLO & $11.2958(4)_{-2.0 \%}^{+2.5 \%}$ & $19.8454(7)_{-2.1 \%}^{+2.5 \%}$ & $0 \%$ & $0 \%$ \\
\hline \multirow[t]{2}{*}{$q \bar{q} \mathrm{NNLO}$} & $12.09(2)_{-1.1 \%}^{+1.1 \%}$ & $21.54(2)_{-1.2 \%}^{+1.1 \%}$ & $+7.0 \%$ & $+8.6 \%$ \\
\hline & \multicolumn{2}{|c|}{$\sigma[\mathrm{fb}]$} & \multicolumn{2}{|c|}{$\sigma / \sigma_{\mathrm{ggLO}}-1$} \\
\hline$g g \mathrm{LO}$ & $0.79355(6)_{-20.9 \%}^{+28.2 \%}$ & $2.0052(1)_{-17.9 \%}^{+23.5 \%}$ & $0 \%$ & $0 \%$ \\
\hline$g g \mathrm{NLO}_{g g}$ & $1.4787(4)_{-13.1 \%}^{+15.9 \%}$ & $3.626(1)_{-12.7 \%}^{+15.2 \%}$ & $+86.3 \%$ & $+80.8 \%$ \\
\hline \multirow[t]{2}{*}{$g g \mathrm{NLO}$} & $1.3892(4)_{-13.6 \%}^{+15.4 \%}$ & $3.425(1)_{-12.0 \%}^{+13.9 \%}$ & $+75.1 \%$ & $+70.8 \%$ \\
\hline & \multicolumn{2}{|c|}{$\sigma[\mathrm{fb}]$} & \multicolumn{2}{|c|}{$\sigma / \sigma_{\mathrm{NLO}}-1$} \\
\hline NNLO & $12.88(2)_{-2.2 \%}^{+2.8 \%}$ & $23.55(2)_{-2.6 \%}^{+3.0 \%}$ & $+14.0 \%$ & $+18.7 \%$ \\
\hline nNNLO & $13.48(2)_{-2.3 \%}^{+2.6 \%}$ & $24.97(2)_{-2.7 \%}^{+2.9 \%}$ & $+19.3 \%$ & $+25.8 \%$ \\
\hline
\end{tabular}

Table 3. Fiducial cross sections at different perturbative orders and relative impact on NLO and $g g \mathrm{LO}$ predictions, respectively. The quoted uncertainties correspond to scale variations as described in the text, and the numerical integration errors on the previous digit are stated in parentheses; for all (n)NNLO results, the latter include the uncertainty due the $r_{\text {cut }}$ extrapolation [52].

NNLO result by about $5 \%(6 \%)$ at $\sqrt{s}=8(13) \mathrm{TeV}$. Corresponding to the above-mentioned numbers, excluding the $q g$ channels would increase the nNNLO prediction by about $1 \%$. The NNLO and nNNLO predictions are marginally compatible within scale uncertainties.

We add a comment on the contribution of diagrams with a Higgs boson: the cuts we are applying essentially select on-shell $Z$ bosons, thereby forcing the Higgs boson to be off-shell. Nonetheless, our calculation consistently includes also the Higgs diagrams. The signal-background interference in the $g g \rightarrow Z Z \rightarrow 4 l$ channel is known to provide a nonnegligible contribution [34]. Indeed, we find that with our selection cuts the impact of the Higgs contribution is about $-5 \%$ both in the $g g \mathrm{LO}$ and $g g \mathrm{NLO}$ results.

We now turn to presenting kinematical distributions. Throughout this section, the plots are organized according to the following pattern: there is an upper panel where absolute cross sections at LO (black, dotted), NLO (red, dashed), NNLO (blue, dashdotted) and nNNLO (magenta, solid) are shown. In the central panel the nNNLO result with its scale uncertainty is normalised to the central NNLO result. In the lower panel the NLO/LO $K$-factors of the loop-induced gluon fusion contribution are shown, with ( $g g \mathrm{NLO}$; pink, solid) and without $\left(g g \mathrm{NLO}_{g g}\right.$; brown, dash-double-dotted) the $q g$ contribution. The figures on the left show the $8 \mathrm{TeV}$ results, and the ones on the right the $13 \mathrm{TeV}$ results.

We first consider the invariant-mass distribution of the four-lepton system in figure 4 . The impact of the NLO corrections to the loop-induced gluon fusion contribution is largest 

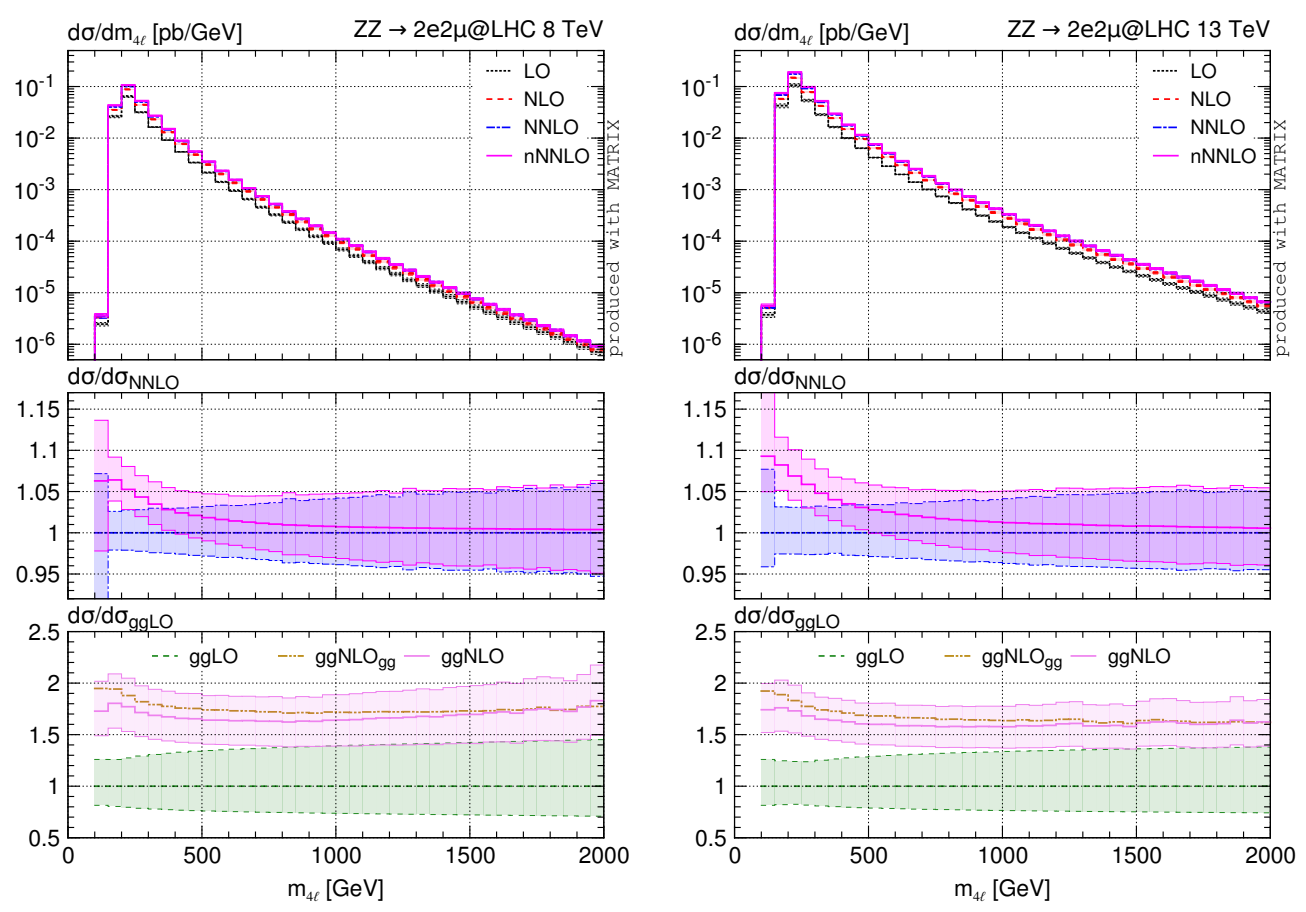

Figure 4. Differential distribution in $m_{4 \ell}$ at $8 \mathrm{TeV}$ (left) and $13 \mathrm{TeV}$ (right).

at small invariant masses: in the peak region they increase the NNLO cross section by about $5 \%(7 \%)$ at $\sqrt{s}=8(13) \mathrm{TeV}$. As $m_{4 \ell}$ increases, the impact of the $g g$ NLO corrections decreases, and it is only about $+1 \%$ at $m_{4 \ell} \sim 1 \mathrm{TeV}$. This is not unexpected, since the $g g$ contribution is largest when gluons with smaller $x$ are probed. On the contrary, the size of the $g g \mathrm{NLO} / g g \mathrm{LO} K$-factor in the lower panel is relatively stable, with a moderate increase at small $m_{4 \ell}$. In both cases, comparing the nNNLO/NNLO and $g g \mathrm{NLO} / g g \mathrm{LO}$ ratios, the scale uncertainties do not fully cover the size of higher-order corrections in the peak region of the distribution, which demonstrates the importance of the NLO corrections to the loopinduced gluon fusion contribution. The impact of the $q g$ channels on the $g g \mathrm{NLO} / g g \mathrm{LO}$ $K$-factor is about $-10 \%$ at smaller $m_{4 \ell}$ values, but essentially vanishes in the tail of the $m_{4 \ell}$ distribution.

In figure 5 we show the invariant-mass distribution of the primary (upper plots) and secondary OSSF lepton pair (lower plots), ordered by the distance of their invariant masses to the $Z$-boson mass. Both distributions are limited by the $Z$-mass window cut in the fiducial phase space. The distribution of the lepton pair which is less close to $m_{Z}$ is broader. More precisely, when the invariant mass of the lepton pair is $m_{Z} \pm 20 \mathrm{GeV}$, the cross section is suppressed by about four and two orders of magnitude for the primary and secondary lepton pair, respectively. Nonetheless, the impact of QCD corrections is uniform in both cases, and independent of the collider energy. The NNLO uncertainty bands barely overlap with the ones of the nNNLO result. 



Figure 5. Invariant-mass distribution of the OSSF lepton pair closer to (top) and further away from (bottom) the $Z$-boson mass at $8 \mathrm{TeV}$ (left) and $13 \mathrm{TeV}$ (right). 

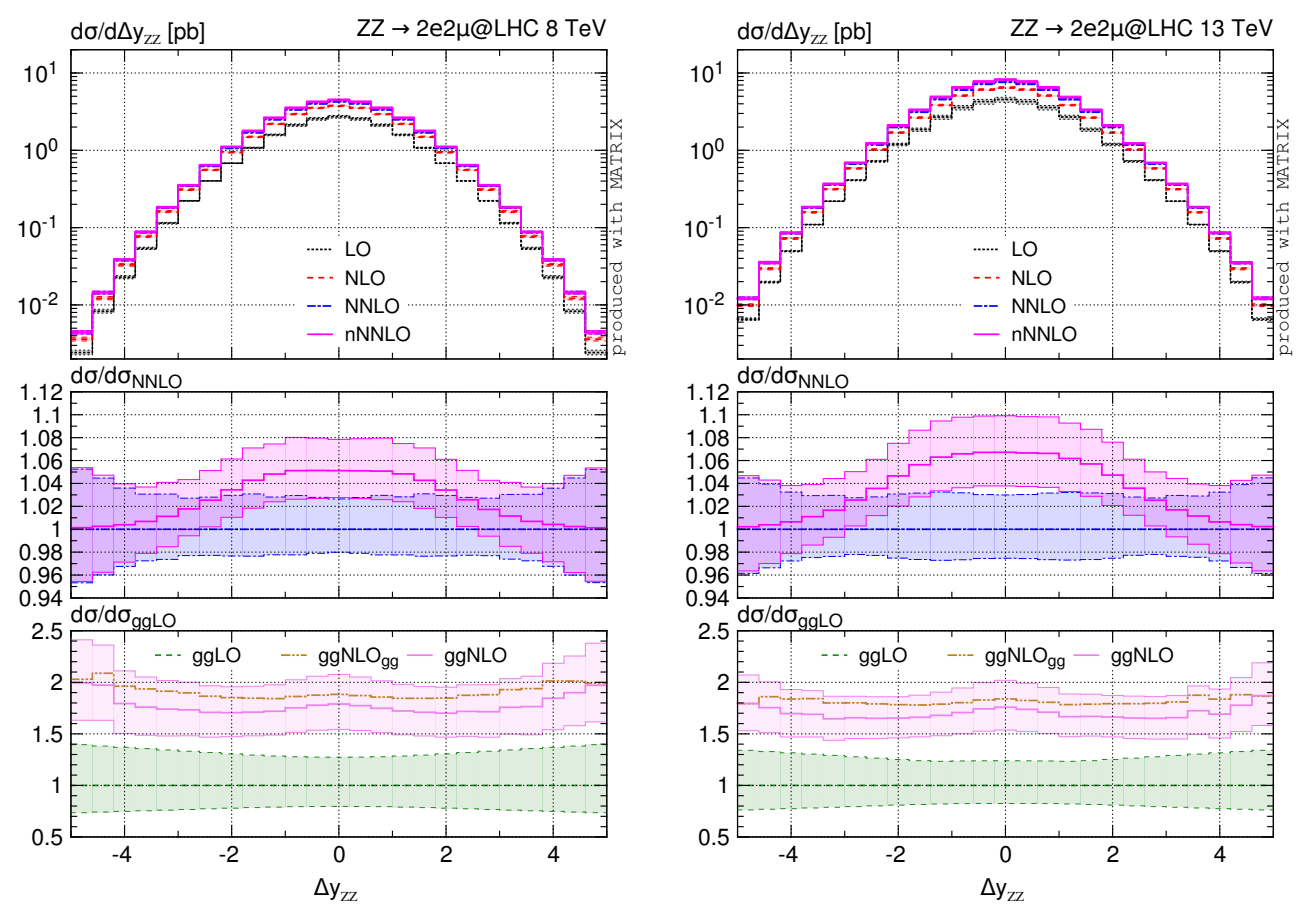

Figure 6. Differential distribution in $\Delta y_{Z Z}$ at $8 \mathrm{TeV}$ (left) and $13 \mathrm{TeV}$ (right).
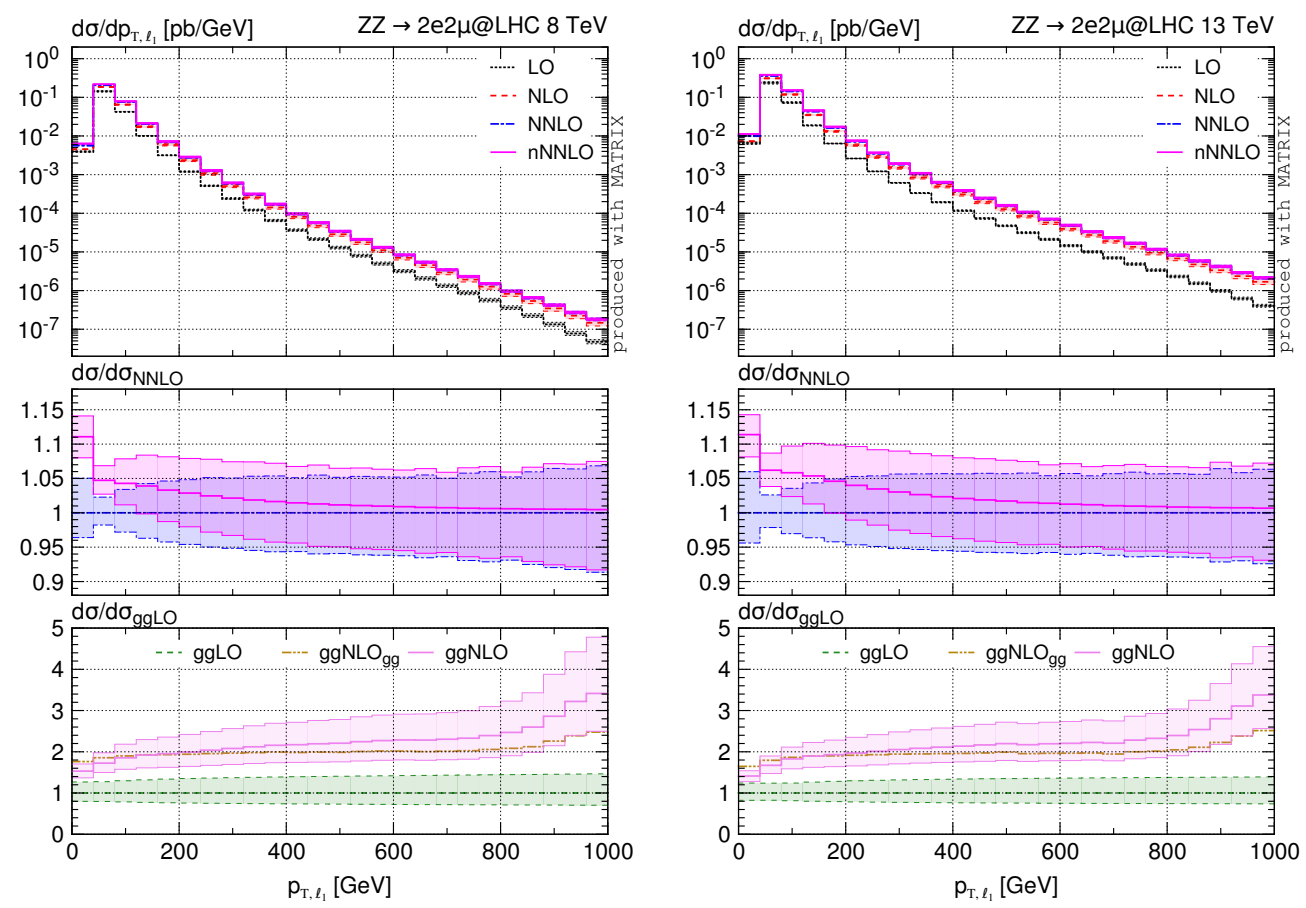

Figure 7. Differential distribution in $p_{T, \ell_{1}}$ at $8 \mathrm{TeV}$ (left) and $13 \mathrm{TeV}$ (right). 
Figure 6 depicts the distribution in the rapidity separation of the two $Z$ bosons $\left(\Delta y_{Z Z}\right)$. The region of small rapidity separations, $\left|\Delta y_{Z Z}\right| \lesssim 1$, is driven by centrally produced $Z$ bosons and thus relatively small partonic momentum fractions, which implies that the relative impact of the gluon fusion contribution is most important there. In this region the impact of the nNNLO corrections is quite uniform and of the order of $+5 \%(+7 \%)$ for $\sqrt{s}=$ 8 (13) TeV, whereas it successively decreases in the forward region. The $g g \mathrm{NLO} / g g \mathrm{LO} K$ factor is quite flat in $\Delta y_{Z Z}$, and also the relative size of the $q g$ contributions is rather uniform over $\Delta y_{Z Z}$.

In figure 7 we study the transverse-momentum distribution of the leading lepton $\left(p_{T, \ell_{1}}\right)$. Analogously to figure 4 , the nNNLO corrections are maximal at small $p_{T, \ell_{1}}$, and they decrease with the value of $p_{T, \ell_{1}}$ : they are about $+5 \%$ in the peak region and drop to about $+1 \%$ at $p_{T, \ell_{1}} \sim 500 \mathrm{GeV}$. Also here the perturbative uncertainties in the first bins do not cover the difference between the NNLO and nNNLO predictions. In contrast to figure 4, the $g g \mathrm{NLO} / g g \mathrm{LO} K$-factor of the gluon fusion contribution becomes larger with the value of $p_{T, \ell_{1}}$, from about 1.7 in the peak region to about 2.2 at $p_{T, \ell_{1}} \sim 500 \mathrm{GeV}$, and it further increases for larger $p_{T, \ell_{1}}$ values. It is interesting to notice that the impact of the newly included $q g$ channels on the $g g \mathrm{NLO}$ corrections depends on the value of $p_{T, \ell_{1}}$, with roughly $-10 \%$ in the peak region and $+10 \%$ for $p_{T, \ell_{1}} \sim 500 \mathrm{GeV}$, thereby affecting the shape of the distribution.

In figure 8 the transverse-momentum distributions of the leading $\left(p_{T, Z_{1}}\right)$ and subleading $\left(p_{T, Z_{2}}\right)$ reconstructed $Z$ bosons are shown. The most prominent feature we notice are the large NLO/LO corrections to the loop-induced gluon fusion channel in case of the harder $Z$ boson, which significantly increases with $p_{T, Z_{1}}$ : the enhancement is by a factor of about 2.8 at $p_{T}=500 \mathrm{GeV}$. This is due to the fact that the phase-space region with one hard $Z$ boson is dominantly populated by events with a jet recoiling against this $Z$ boson, while the other $Z$ boson is relatively soft. Such large corrections are absent in the $p_{T, Z_{2}}$ distribution: the phase-space region where both $Z$ bosons have large transverse momenta is naturally dominated by topologies with the two $Z$ bosons recoiling against each other, which are already present at LO and thus do not give rise to exceptionally large corrections. This situation also explains the opposite behaviour of the NLO/LO $K$-factor in $p_{T, Z_{2}}$ which continuously decreases with $p_{T, Z_{2}}$. The previous statements are not specific to the loopinduced gluon fusion channel: we observe the same features also for the NLO corrections to the quark annihilation channel.

Also for the transverse-momentum distributions of the $Z$ bosons the importance of the $q g$ channels in the $g g$ NLO result is evident: the $p_{T, Z_{1}}$ shape is clearly modified due to a negative $q g$ contribution at small $p_{T, Z_{1}}$, and a positive $q g$ contribution in the tail of the distribution. At large $p_{T, Z_{2}}$ the contribution of the $q g$ channels is as large as the one of the $g g$ channel. However, they have opposite signs such that they compensate each other and the $g g \mathrm{NLO}$ corrections almost vanish, whereas, neglecting $q g$ contributions, the $g g \mathrm{NLO}_{g g}$ corrections show an increase of roughly $40 \%$ wrt. $g g$ LO instead. NNLO scale uncertainties at small $p_{T, Z_{1}}$ and $p_{T, Z_{2}}$ typically do not cover the sizeable nNNLO corrections.

Another eye-catching feature we observe in figure 8 is the significant drop of the transverse-momentum distribution of both the leading and subleading $Z$ boson above 

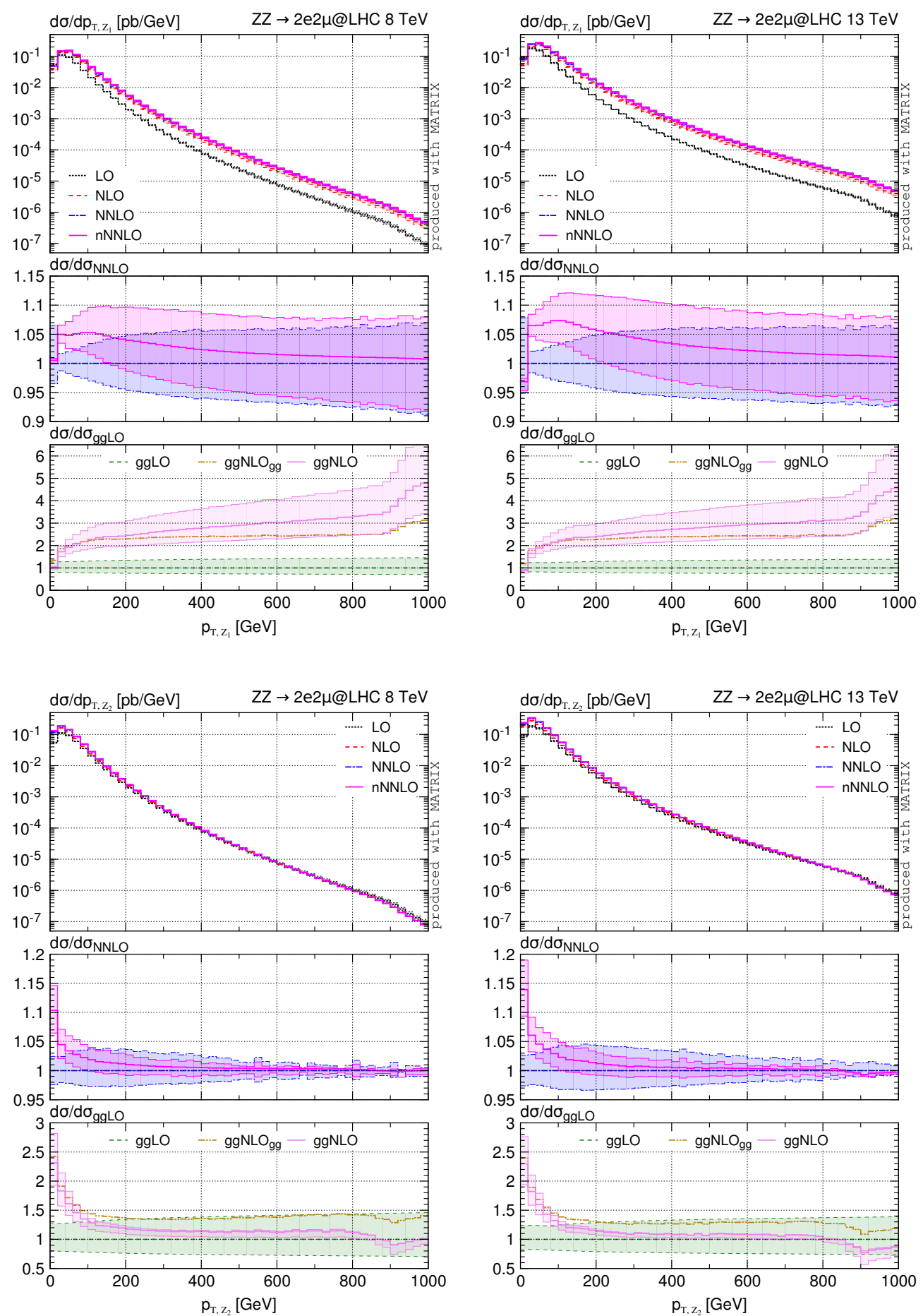

Figure 8. Transverse-momentum spectra of the leading (top) and subleading (bottom) $Z$ boson at $8 \mathrm{TeV}$ (left) and $13 \mathrm{TeV}$ (right). 
$p_{T, Z_{i}} \sim 900 \mathrm{GeV}(i \in\{1,2\})$. This is due to the interplay between the large transverse momentum of the parent $Z$ boson, which makes the corresponding lepton pair boosted, and the $\Delta R_{\ell \ell}>\Delta R_{\ell \ell}^{\min }$ cut in the fiducial phase space $\left(\ell \in\{e, \mu\}, \Delta R_{\ell \ell}^{\min }=0.2\right)$. Indeed, if the transverse momentum of the parent $Z$ boson fulfills the condition

$$
p_{T, Z_{i}} \gtrsim \frac{\sqrt{2} m_{Z}}{\sqrt{1-\cos \Delta R_{\ell \ell}^{\min }}} \sim 900 \mathrm{GeV},
$$

the lepton pair is forced to be produced off-shell, and as a consequence the cross section is strongly suppressed. Note that this effect is independent of the collider energy.

\section{Summary}

We have calculated the NLO QCD corrections to the loop-induced gluon fusion contribution for $Z Z$ production in the four-lepton channel. Our predictions include, for the first time, also (anti)quark-gluon partonic channels. We have combined these results with state-of-theart NNLO QCD corrections to the quark annihilation channel, yielding an approximation of the full $\mathrm{N}^{3} \mathrm{LO}$ QCD corrections for $Z Z$ production, denoted by nNNLO.

We have performed an extensive validation against existing results for the NLO cross section of the loop-induced gluon fusion contribution in the literature [33, 35]. Overall, we find decent agreement of the total rates and distributions by adopting the respective setups, thereby neglecting contributions from $q g$ channels and massive top-quark loops.

We have presented a comprehensive study of the nNNLO corrections, the size of the $g g \mathrm{NLO} / g g \mathrm{LO} K$-factors of the loop-induced gluon fusion contribution, and the impact of the newly computed $q g$ channels. The main conclusions can be summarized as follows:

- The loop-induced gluon fusion contribution is sizeable. It makes up roughly $57 \%(62 \%)$ of the full $\mathcal{O}\left(\alpha_{\mathrm{S}}^{2}\right)$ corrections to $Z Z$ production, and yields roughly $6 \%(9 \%)$ of the NNLO cross section at $8(13) \mathrm{TeV}$ collider energy. Hence, its NLO QCD corrections are important, with $K$-factors of $\gtrsim 1.7$ wrt. $g g \mathrm{LO}$ : the nNNLO cross section is about $5 \%(6 \%)$ larger than the NNLO one at $\sqrt{s}=8(13) \mathrm{TeV}$, and their perturbative uncertainties barely overlap.

- The $q g$ channels have a negative effect of about $10 \%$ on the $g g \mathrm{NLO} / g g \mathrm{LO} K$-factor of the loop-induced gluon fusion contribution, which yields roughly a $1 \%$ decrease of the nNNLO cross section.

- The nNNLO corrections can have a non-trivial impact on differential distributions. Due to the nature of the loop-induced gluon fusion production mechanism, and the dominance of the gluon densities at small $x$, the $\mathrm{nNNLO}$ corrections provide sizeable effects in dominant phase-space regions of the observables we investigated: at small invariant-masses of the four lepton system, in the central region of the rapidity difference between the two reconstructed $Z$ bosons, and at small transverse momenta of the leptons and $Z$ bosons. 
- In these regions, where the computed corrections are largest, nNNLO and NNLO scale-uncertainty bands barely overlap, which demonstrates the importance of including the NLO QCD corrections to the loop-induced gluon fusion contribution.

- For the transverse-momentum distributions of the leptons and the reconstructed $Z$ bosons the newly computed contributions of the $q g$ channels have a significant impact on the shapes of the $g g \mathrm{NLO}$ spectra.

The NLO QCD corrections to the loop-induced gluon fusion contribution have been implemented in our parton-level Monte Carlo code MATRIX to provide nNNLO cross sections for charge-neutral diboson production processes. The consistent combination of state-ofthe-art predictions for quark annihilation and loop-induced gluon fusion production mechanisms within a single tool may turn out to be particularly useful for the experimental analyses. MATRIX can be used not only to obtain the best QCD prediction for diboson cross sections, but also to estimate perturbative uncertainties consistently from simultaneous scale variations within the two contributions. The inclusion of NLO EW corrections in these calculations to obtain ultimate perturbative accuracy in diboson predictions is left for future work.

\section{Acknowledgments}

We thank Simone Alioli, Fabrizio Caola, and Lorenzo Tancredi for helpful discussions, and for providing details on their computations and results. We are particularly indebted to Jonas Lindert for making private OpenLoops amplitudes for the loop-induced channel available to us, notably spin and colour correlators and several approximations with and without top-quark contributions, and Simone Alioli for private communication of the statistical uncertainties to the results of ref. [35]. Moreover, we want to thank Jean-Nicolas Lang for his help on interfacing RECOLA and validating the OpEnLoOps results. This work is supported in part by the Swiss National Science Foundation (SNF) under contracts CRSII2141847 and 200020-169041. The work of MW is supported by the ERC Consolidator Grant 614577 HICCUP, and that of SK by the ERC Starting Grant 714788 REINVENT.

Open Access. This article is distributed under the terms of the Creative Commons Attribution License (CC-BY 4.0), which permits any use, distribution and reproduction in any medium, provided the original author(s) and source are credited.

\section{References}

[1] ATLAS collaboration, Observation of a new particle in the search for the Standard Model Higgs boson with the ATLAS detector at the LHC, Phys. Lett. B 716 (2012) 1 [arXiv: 1207.7214] [INSPIRE].

[2] CMS collaboration, Observation of a new boson at a mass of $125 \mathrm{GeV}$ with the CMS experiment at the LHC, Phys. Lett. B 716 (2012) 30 [arXiv:1207.7235] [INSPIRE].

[3] ATLAS collaboration, Evidence for the spin-0 nature of the Higgs boson using ATLAS data, Phys. Lett. B 726 (2013) 120 [arXiv:1307.1432] [INSPIRE]. 
[4] CMS collaboration, Measurement of the properties of a Higgs boson in the four-lepton final state, Phys. Rev. D 89 (2014) 092007 [arXiv: 1312.5353] [INSPIRE].

[5] CMS collaboration, Limits on the Higgs boson lifetime and width from its decay to four charged leptons, Phys. Rev. D 92 (2015) 072010 [arXiv:1507.06656] [INSPIRE].

[6] ATLAS collaboration, Constraints on off-shell Higgs boson production and the Higgs boson total width in $Z Z \rightarrow 4 \ell$ and $Z Z \rightarrow 2 \ell 2 \nu$ final states with the ATLAS detector, Phys. Lett. B 786 (2018) 223 [arXiv: 1808.01191] [INSPIRE].

[7] ATLAS and CMS collaborations, Measurements of the Higgs boson production and decay rates and constraints on its couplings from a combined ATLAS and CMS analysis of the LHC pp collision data at $\sqrt{s}=7$ and $8 \mathrm{TeV}$, JHEP 08 (2016) 045 [arXiv:1606.02266] [INSPIRE].

[8] J. Ohnemus and J.F. Owens, An order $\alpha_{s}$ calculation of hadronic ZZ production, Phys. Rev. D 43 (1991) 3626 [INSPIRE].

[9] B. Mele, P. Nason and G. Ridolfi, QCD radiative corrections to $Z$ boson pair production in hadronic collisions, Nucl. Phys. B 357 (1991) 409 [INSPIRE].

[10] J. Ohnemus, Hadronic $Z Z, W^{-} W^{+}$and $W^{ \pm} Z$ production with $Q C D$ corrections and leptonic decays, Phys. Rev. D 50 (1994) 1931 [hep-ph/9403331] [INSPIRE].

[11] J.M. Campbell and R.K. Ellis, An update on vector boson pair production at hadron colliders, Phys. Rev. D 60 (1999) 113006 [hep-ph/9905386] [InSPIRE].

[12] L.J. Dixon, Z. Kunszt and A. Signer, Vector boson pair production in hadronic collisions at order $\alpha_{s}$ : lepton correlations and anomalous couplings, Phys. Rev. D 60 (1999) 114037 [hep-ph/9907305] [INSPIRE].

[13] L.J. Dixon, Z. Kunszt and A. Signer, Helicity amplitudes for $O\left(\alpha_{s}\right)$ production of $W^{+} W^{-}$, $W^{ \pm} Z, Z Z, W^{ \pm} \gamma$, or $Z \gamma$ pairs at hadron colliders, Nucl. Phys. B 531 (1998) 3 [hep-ph/9803250] [INSPIRE].

[14] E. Accomando, A. Denner and A. Kaiser, Logarithmic electroweak corrections to gauge-boson pair production at the LHC, Nucl. Phys. B 706 (2005) 325 [hep-ph/0409247] [INSPIRE].

[15] A. Bierweiler, T. Kasprzik and J.H. Kühn, Vector-boson pair production at the LHC to $O\left(\alpha^{3}\right)$ accuracy, JHEP 12 (2013) 071 [arXiv: 1305.5402] [INSPIRE].

[16] J. Baglio, L.D. Ninh and M.M. Weber, Massive gauge boson pair production at the LHC: a next-to-leading order story, Phys. Rev. D 88 (2013) 113005 [Erratum ibid. D 94 (2016) 099902] [arXiv:1307.4331] [INSPIRE].

[17] B. Biedermann, A. Denner, S. Dittmaier, L. Hofer and B. Jäger, Electroweak corrections to $p p \rightarrow \mu^{+} \mu^{-} e^{+} e^{-}+X$ at the LHC: a Higgs background study, Phys. Rev. Lett. 116 (2016) 161803 [arXiv: 1601.07787] [INSPIRE].

[18] B. Biedermann, A. Denner, S. Dittmaier, L. Hofer and B. Jäger, Next-to-leading-order electroweak corrections to the production of four charged leptons at the LHC, JHEP 01 (2017) 033 [arXiv : 1611.05338] [INSPIRE].

[19] S. Kallweit, J.M. Lindert, S. Pozzorini and M. Schönherr, NLO QCD+EW predictions for $2 \ell 2 \nu$ diboson signatures at the LHC, JHEP 11 (2017) 120 [arXiv:1705.00598] [INSPIRE].

[20] M. Chiesa, A. Denner and J.-N. Lang, Anomalous triple-gauge-boson interactions in vector-boson pair production with RECOLA2, Eur. Phys. J. C 78 (2018) 467 [arXiv: 1804.01477] [INSPIRE]. 
[21] T. Binoth, T. Gleisberg, S. Karg, N. Kauer and G. Sanguinetti, NLO QCD corrections to $Z Z+$ jet production at hadron colliders, Phys. Lett. B 683 (2010) 154 [arXiv:0911.3181] [INSPIRE].

[22] E.W.N. Glover and J.J. van der Bij, $Z$ boson pair production via gluon fusion, Nucl. Phys. B 321 (1989) 561 [INSPIRE].

[23] D.A. Dicus, C. Kao and W.W. Repko, Gluon production of gauge bosons, Phys. Rev. D 36 (1987) 1570 [INSPIRE].

[24] T. Matsuura and J.J. van der Bij, Characteristics of leptonic signals for $Z$ boson pairs at hadron colliders, Z. Phys. C 51 (1991) 259 [INSPIRE].

[25] C. Zecher, T. Matsuura and J.J. van der Bij, Leptonic signals from off-shell Z boson pairs at hadron colliders, Z. Phys. C 64 (1994) 219 [hep-ph/9404295] [INSPIRE].

[26] T. Binoth, N. Kauer and P. Mertsch, Gluon-induced QCD corrections to $p p \rightarrow Z Z \rightarrow \ell \bar{\ell} \ell^{\prime} \bar{\ell}^{\prime}$, in Proceedings, $16^{\text {th }}$ International Workshop on Deep Inelastic Scattering and Related Subjects (DIS 2008), London, U.K. 7-11 April 2008, pg. 142 [arXiv:0807.0024] [InSPIRE].

[27] J.M. Campbell, R.K. Ellis and C. Williams, Vector boson pair production at the LHC, JHEP 07 (2011) 018 [arXiv: 1105.0020] [INSPIRE].

[28] N. Kauer, Interference effects for $H \rightarrow W W / Z Z \rightarrow \ell \bar{\nu}_{\ell} \bar{\ell} \nu_{\ell}$ searches in gluon fusion at the LHC, JHEP 12 (2013) 082 [arXiv:1310.7011] [InSPIRE].

[29] F. Cascioli, S. Höche, F. Krauss, P. Maierhöfer, S. Pozzorini and F. Siegert, Precise Higgs-background predictions: merging NLO QCD and squared quark-loop corrections to four-lepton + 0,1 jet production, JHEP 01 (2014) 046 [arXiv:1309.0500] [INSPIRE].

[30] J.M. Campbell, R.K. Ellis and C. Williams, Bounding the Higgs width at the LHC using full analytic results for $g g \rightarrow e^{-} e^{+} \mu^{-} \mu^{+}$, JHEP 04 (2014) 060 [arXiv: 1311.3589] [INSPIRE].

[31] J.M. Campbell, R.K. Ellis and C. Williams, Bounding the Higgs width at the LHC, PoS (LL2014) 008 (2014) [arXiv: 1408.1723] [INSPIRE].

[32] N. Kauer, C. O'Brien and E. Vryonidou, Interference effects for $H \rightarrow W W \rightarrow \ell \nu q \bar{q}^{\prime}$ and $H \rightarrow Z Z \rightarrow \ell \bar{\ell} q \bar{q}$ searches in gluon fusion at the LHC, JHEP 10 (2015) 074 [arXiv: 1506.01694] [INSPIRE].

[33] F. Caola, K. Melnikov, R. Röntsch and L. Tancredi, QCD corrections to ZZ production in gluon fusion at the LHC, Phys. Rev. D 92 (2015) 094028 [arXiv:1509.06734] [INSPIRE].

[34] F. Caola, M. Dowling, K. Melnikov, R. Röntsch and L. Tancredi, QCD corrections to vector boson pair production in gluon fusion including interference effects with off-shell Higgs at the LHC, JHEP 07 (2016) 087 [arXiv: 1605.04610] [INSPIRE].

[35] S. Alioli, F. Caola, G. Luisoni and R. Röntsch, ZZ production in gluon fusion at NLO matched to parton-shower, Phys. Rev. D 95 (2017) 034042 [arXiv:1609.09719] [InSPIRE].

[36] F. Caola, J.M. Henn, K. Melnikov, A.V. Smirnov and V.A. Smirnov, Two-loop helicity amplitudes for the production of two off-shell electroweak bosons in gluon fusion, JHEP 06 (2015) 129 [arXiv: 1503.08759] [INSPIRE].

[37] A. von Manteuffel and L. Tancredi, The two-loop helicity amplitudes for $g g \rightarrow V_{1} V_{2} \rightarrow 4$ leptons, JHEP 06 (2015) 197 [arXiv: 1503.08835] [INSPIRE].

[38] F. Cascioli et al., ZZ production at hadron colliders in NNLO QCD, Phys. Lett. B 735 (2014) 311 [arXiv:1405.2219] [INSPIRE]. 
[39] G. Heinrich, S. Jahn, S.P. Jones, M. Kerner and J. Pires, NNLO predictions for Z-boson pair production at the LHC, JHEP 03 (2018) 142 [arXiv:1710.06294] [INSPIRE].

[40] T. Gehrmann, A. von Manteuffel, L. Tancredi and E. Weihs, The two-loop master integrals for $q \bar{q} \rightarrow V V$, JHEP 06 (2014) 032 [arXiv: 1404.4853] [INSPIRE].

[41] F. Caola, J.M. Henn, K. Melnikov, A.V. Smirnov and V.A. Smirnov, Two-loop helicity amplitudes for the production of two off-shell electroweak bosons in quark-antiquark collisions, JHEP 11 (2014) 041 [arXiv:1408.6409] [INSPIRE].

[42] T. Gehrmann, A. von Manteuffel and L. Tancredi, The two-loop helicity amplitudes for $q \bar{q}^{\prime} \rightarrow V_{1} V_{2} \rightarrow 4$ leptons, JHEP 09 (2015) 128 [arXiv:1503.04812] [INSPIRE].

[43] M. Grazzini, S. Kallweit and D. Rathlev, ZZ production at the LHC: fiducial cross sections and distributions in NNLO QCD, Phys. Lett. B 750 (2015) 407 [arXiv:1507.06257] [INSPIRE].

[44] S. Kallweit and M. Wiesemann, $Z Z$ production at the $L H C$ : NNLO predictions for $2 \ell 2 \nu$ and $4 \ell$ signatures, Phys. Lett. B 786 (2018) 382 [arXiv: 1806.05941] [INSPIRE].

[45] T. Gehrmann et al., $W^{+} W^{-}$production at hadron colliders in next to next to leading order QCD, Phys. Rev. Lett. 113 (2014) 212001 [arXiv:1408.5243] [INSPIRE].

[46] M. Grazzini, S. Kallweit, S. Pozzorini, D. Rathlev and M. Wiesemann, $W^{+} W^{-}$production at the LHC: fiducial cross sections and distributions in NNLO QCD, JHEP 08 (2016) 140 [arXiv: 1605.02716] [INSPIRE].

[47] F. Caola, K. Melnikov, R. Röntsch and L. Tancredi, QCD corrections to $W^{+} W^{-}$production through gluon fusion, Phys. Lett. B 754 (2016) 275 [arXiv:1511.08617] [INSPIRE].

[48] ATLAS collaboration, $Z Z \rightarrow \ell^{+} \ell^{-} \ell^{\prime} \ell^{\prime-}$ cross-section measurements and search for anomalous triple gauge couplings in $13 \mathrm{TeV}$ pp collisions with the ATLAS detector, Phys. Rev. D 97 (2018) 032005 [arXiv: 1709.07703] [INSPIRE].

[49] ATLAS collaboration, Measurement of the $W^{+} W^{-}$production cross section in pp collisions at a centre-of-mass energy of $\sqrt{s}=13 \mathrm{TeV}$ with the ATLAS experiment, Phys. Lett. B 773 (2017) 354 [arXiv: 1702. 04519] [INSPIRE].

[50] CMS collaboration, Measurements of the $p p \rightarrow Z Z$ production cross section and the $Z \rightarrow 4 \ell$ branching fraction and constraints on anomalous triple gauge couplings at $\sqrt{s}=13 \mathrm{TeV}$, Eur. Phys. J. C 78 (2018) 165 [Erratum ibid. C 78 (2018) 515] [arXiv:1709.08601] [INSPIRE].

[51] CMS collaboration, Measurement of the $W W$ cross section pp collisions at $\sqrt{s}=13 \mathrm{TeV}$, CMS-PAS-SMP-16-006, CERN, Geneva, Switzerland (2016).

[52] M. Grazzini, S. Kallweit and M. Wiesemann, Fully differential NNLO computations with MATRIX, Eur. Phys. J. C 78 (2018) 537 [arXiv:1711.06631] [InSPIRE].

[53] S. Catani and M. Grazzini, An NNLO subtraction formalism in hadron collisions and its application to Higgs boson production at the LHC, Phys. Rev. Lett. 98 (2007) 222002 [hep-ph/0703012] [INSPIRE].

[54] M. Grazzini, S. Kallweit, D. Rathlev and A. Torre, $Z \gamma$ production at hadron colliders in NNLO QCD, Phys. Lett. B 731 (2014) 204 [arXiv:1309.7000] [INSPIRE].

[55] M. Grazzini, S. Kallweit and D. Rathlev, $W \gamma$ and $Z \gamma$ production at the LHC in NNLO QCD, JHEP 07 (2015) 085 [arXiv: 1504.01330] [INSPIRE]. 
[56] M. Grazzini, S. Kallweit, D. Rathlev and M. Wiesemann, $W^{ \pm} Z$ production at hadron colliders in NNLO QCD, Phys. Lett. B 761 (2016) 179 [arXiv:1604.08576] [INSPIRE].

[57] M. Grazzini, S. Kallweit, D. Rathlev and M. Wiesemann, $W^{ \pm} Z$ production at the LHC: fiducial cross sections and distributions in NNLO QCD, JHEP 05 (2017) 139 [arXiv: 1703.09065] [INSPIRE].

[58] D. de Florian et al., Differential Higgs boson pair production at next-to-next-to-leading order in QCD, JHEP 09 (2016) 151 [arXiv: 1606.09519] [INSPIRE].

[59] M. Grazzini et al., Higgs boson pair production at NNLO with top quark mass effects, JHEP 05 (2018) 059 [arXiv: 1803. 02463] [INSPIRE].

[60] M. Grazzini, S. Kallweit, D. Rathlev and M. Wiesemann, Transverse-momentum resummation for vector-boson pair production at NNLL+NNLO, JHEP 08 (2015) 154 [arXiv: 1507.02565] [INSPIRE].

[61] E. Re, M. Wiesemann and G. Zanderighi, NNLOPS accurate predictions for $W^{+} W^{-}$ production, JHEP 12 (2018) 121 [arXiv:1805.09857] [INSPIRE].

[62] S. Kallweit, J.M. Lindert, P. Maierhöfer, S. Pozzorini and M. Schönherr, NLO electroweak automation and precise predictions for $W+$ multijet production at the LHC, JHEP 04 (2015) 012 [arXiv:1412.5157] [INSPIRE].

[63] S. Kallweit, J.M. Lindert, P. Maierhöfer, S. Pozzorini and M. Schönherr, $N L O Q C D+E W$ predictions for $V+$ jets including off-shell vector-boson decays and multijet merging, JHEP 04 (2016) 021 [arXiv: 1511.08692] [INSPIRE].

[64] S. Kallweit, MUlti-chaNnel Integrator at Swiss (CH) precision - an automated parton level $N L O$ generator, in preparation.

[65] A. Denner, S. Dittmaier and L. Hofer, COLLIER - a fortran-library for one-loop integrals, PoS (LL2014) 071 (2014) [arXiv: 1407.0087] [INSPIRE].

[66] A. Denner, S. Dittmaier and L. Hofer, COLLIER: a fortran-based Complex One-Loop LIbrary in Extended Regularizations, Comput. Phys. Commun. 212 (2017) 220 [arXiv: 1604.06792] [INSPIRE].

[67] G. Ossola, C.G. Papadopoulos and R. Pittau, CutTools: a program implementing the OPP reduction method to compute one-loop amplitudes, JHEP 03 (2008) 042 [arXiv:0711.3596] [INSPIRE].

[68] A. van Hameren, OneLOop: for the evaluation of one-loop scalar functions, Comput. Phys. Commun. 182 (2011) 2427 [arXiv:1007.4716] [INSPIRE].

[69] F. Buccioni, J.M. Lindert, J.-N. Lang, P. Maierhöfer, S. Pozzorini, H. Zhang and M. Zoller, OpenLoops 2.0, in preparation.

[70] F. Cascioli, P. Maierhöfer and S. Pozzorini, Scattering amplitudes with open loops, Phys. Rev. Lett. 108 (2012) 111601 [arXiv:1111.5206] [INSPIRE].

[71] F. Buccioni, S. Pozzorini and M. Zoller, On-the-fly reduction of open loops, Eur. Phys. J. C 78 (2018) 70 [arXiv:1710.11452] [INSPIRE].

[72] S. Actis, A. Denner, L. Hofer, J.-N. Lang, A. Scharf and S. Uccirati, RECOLA: rEcursive Computation of One-Loop Amplitudes, Comput. Phys. Commun. 214 (2017) 140 [arXiv: 1605.01090] [INSPIRE]. 
[73] A. Denner, J.-N. Lang and S. Uccirati, Recola2: REcursive Computation of One-Loop Amplitudes 2, Comput. Phys. Commun. 224 (2018) 346 [arXiv: 1711.07388] [INSPIRE].

[74] S. Catani and M.H. Seymour, The dipole formalism for the calculation of QCD jet cross-sections at next-to-leading order, Phys. Lett. B 378 (1996) 287 [hep-ph/9602277] [INSPIRE].

[75] S. Catani and M.H. Seymour, A general algorithm for calculating jet cross-sections in NLO QCD, Nucl. Phys. B 485 (1997) 291 [Erratum ibid. B 510 (1998) 503] [hep-ph/9605323] [INSPIRE].

[76] NNPDF collaboration, Parton distributions for the LHC run II, JHEP 04 (2015) 040 [arXiv: 1410.8849$]$ [INSPIRE].

[77] A. Denner, S. Dittmaier, M. Roth and L.H. Wieders, Electroweak corrections to charged-current $e^{+} e^{-} \rightarrow 4$ fermion processes: technical details and further results, Nucl. Phys. B 724 (2005) 247 [Erratum ibid. B 854 (2012) 504] [hep-ph/0505042] [INSPIRE].

[78] Particle Data Group collaboration, Review of particle physics, Chin. Phys. C 40 (2016) 100001 [INSPIRE].

[79] ATLAS collaboration, Measurement of the $Z Z$ production cross section in proton-proton collisions at $\sqrt{s}=8 \mathrm{TeV}$ using the $Z Z \rightarrow \ell^{-} \ell^{+} \ell^{\prime} \ell^{\prime+}$ and $Z Z \rightarrow \ell^{-} \ell^{+} \nu \bar{\nu}$ channels with the ATLAS detector, JHEP 01 (2017) 099 [arXiv:1610.07585] [INSPIRE]. 